\title{
A mechanism for streamwise localisation of nonlinear waves in shear flows
}

\author{
Fernando Mellibovsky ${ }^{1} \dagger$, and Alvaro Meseguer ${ }^{2}$ \\ ${ }^{1}$ Castelldefels School of Telecom and Aerospace Engineering, Universitat Politècnica de Catalunya, \\ 08034, Barcelona, Spain \\ ${ }^{2}$ Departament de Física Aplicada, Universitat Politècnica de Catalunya, 08034, Barcelona, Spain
}

(Received xx; revised xx; accepted xx)

\begin{abstract}
We present the complete unfolding of streamwise localisation in a paradigm of extended shear flows, namely, two-dimensional plane-Poiseuille flow. Exact solutions of the Navier-Stokes equations are computed numerically and tracked in the streamwise wavenumber - Reynolds number parameter space to identify and describe the fundamental mechanism behind streamwise localisation, a ubiquitous feature of shear flow turbulence. Unlike shear flow spanwise localisation, streamwise localisation does not follow the snaking mechanism demonstrated for plane-Couette flow.
\end{abstract}

Key words:

\section{Introduction}

Turbulent flows pervade both nature and engineering applications, with far-reaching implications. Mixing of physical properties, flow homogeneity, friction or flow separation crucially rely on the laminar or turbulent nature of fluid flow. In the case of wall-bounded shear flows, such as those in pipes, channels or boundary layers, turbulence often sets in, for large enough flow rates, despite the linear stability of the laminar state (Reynolds 1883; Eckhardt et al. 2007), and does so in the form of localised patches of turbulent flow (Wygnanski \& Champagne 1973) that only delocalise to take up larger and larger portions of the domain once the flow rate is further increased beyond a critical value (Avila et al. 2011). The mechanism underlying localisation is of paramount importance not only to fluid flows, but also to the comprehension of the physics of nonlinear extended systems (Cross \& Hohenberg 1993).

The coexistence of the laminar and a turbulent state in shear flows has recently been ascribed to the appearance, above a certain value of the flow rate, of pairs of simple solutions of the Navier-Stokes equations in saddle-node bifurcations (Nagata 1990; Faisst \& Eckhardt 2003; Wedin \& Kerswell 2004). Short periodic pipes and channels shown to properly reproduce developed turbulence local dynamics (the minimal flow unit, see e.g. Jimenez 1990; Hamilton et al. 1995), have been extensively used as a proof-of-concept. The saddle solution, together with its stable manifold, has been shown to reside in the laminar-turbulent separatrix and to govern the transitional dynamics (Duguet et al. 2008; Schneider et al. 2008; Itano \& Toh 2000; Kreilos et al. 2013). Meanwhile, the nodal solution, initially presenting little or no time complexity, undergoes a bifurcation cascade that eventually results in a chaotic saddle that is capable of sustaining turbulent dynamics of transient nature (Mellibovsky \& Eckhardt 2012). Fully developed turbulent flow has indeed been shown to rely on the existence of these so-called

$\dagger$ Email address for correspondence: fernando.mellibovsky@upc.edu 
upper branch solutions both numerically (Kerswell \& Tutty 2007) and experimentally (Hof et al. 2004). This scenario has been confirmed for extended domains allowing for localisation, albeit with modifications regarding the spatial structure of the underlying solution, which is no longer periodic but localised (Mellibovsky et al. 2009; Duguet et al. 2009). Fully localised invariant solutions of the Navier-Stokes equations have since been found by restricting the dynamics to appropriate symmetry subspaces in long pipes (Avila et al. 2013), and in extended domains (spanwise and/or streamwise) in plane Couette (Gibson \& Brand 2014; Brand \& Gibson 2014) and plane Poiseuille flows (Zammert \& Eckhardt 2014).

The mechanism behind localisation, a salient feature of transitional dynamics, has only recently been confronted for shear flows. Although other mechanisms might be possible, the only known path to spanwise localisation of channel flow has been proven to result from subharmonic instability of spanwise periodic waves that become localised in a wave packet (Schneider et al. 2010; Melnikov et al. 2014; Chantry et al. 2015) via a snaking mechanism (Burke \& Knobloch 2007b, a; Bergeon et al. 2008; Mercader et al. 2009). Streamwise localisation appears fundamentally different from spanwise localisation. It has been found to also emerge from subharmonic instability of streamwise periodic solutions through domain length continuation of a branch of modulated travelling waves (Chantry et al. 2014), but whether snaking still plays a part remains yet to be addressed.

A complete unfolding of the mechanism underlying streamwise localisation requires an extensive exploration in parameter space including extremely costly continuation of time-dependent nonlinear solutions. Although continuation of fully three-dimensional localised solutions has been shown possible (Chantry et al. 2014), the refined multiparametric study required here is unaffordable for three-dimensional systems such as pipe or channel flows, where the initial steps towards explaining the phenomenon have been undertaken, but becomes feasible for the simplest flow exhibiting archetypal streamwise localisation, ie 2D plane-Poiseuille flow (PPF). This problem gathers all the required ingredients: subcritical bifurcation of upper and lower branch streamwise-periodic solutions (Tollmien-Schlichting waves, Chen \& Joseph (1973); Zahn et al. (1974)), subharmonic bifurcation of localised wave-packet solutions (Jimenez 1990; Drissi et al. 1999) and the advantage of two-dimensionality. In this Rapids we exploit this fact to resolve the problem of streamwise localisation in shear flows.

The outline of the paper is as follows. In $\S 2$, we present plane Poiseuille flow and describe the formulation we adopt. In $\S 3$ we sketch the numerical methods we employ to tackle its resolution. Results are given and discussed in $\S 4$. Some concluding remarks are finally provided in $\S 5$.

\section{Problem formulation}

We consider the pressure-driven incompressible twodimensional (in the $(x, y)$-plane) flow of a Newtonian fluid (density $\rho$ and kinematic viscosity $\nu$ ) between two infinite parallel plates at $y= \pm h$ ( $h$ is half the gapwidth). The flow is governed by the Navier-Stokes equations:

$$
\partial_{t} \mathbf{u}+(\mathbf{u} \cdot \nabla) \mathbf{u}=-\frac{1}{\rho} \nabla p+\nu \nabla^{2} \mathbf{u}-\frac{\Pi}{\rho} \hat{\mathbf{x}}, \quad \nabla \cdot \mathbf{u}=0,
$$

where $\mathbf{u}=(u, v)$ and $p$ are the velocity and pressure fields, respectively, and $\Pi$ is the driving streamwise pressure gradient. The no-slip boundary condition imposes $\mathbf{u}(x, \pm h, t)=(0,0)$ and streamwise periodicity is assumed for both $\mathbf{u}$ and $p$.

PPF (and pipe Poiseuille flow) may be driven by a constant pressure drop along the domain (usual method of choice in experiments: constant $\Pi$ ) or, alternatively, by an instantly adjusting pressure drop that enforces a constant mass flux (implemented in experiments by sucking from the outlet with a constant velocity piston: adjustable $\Pi$ ) (Pugh \& Saffman 1988). Both driving mechanisms are nearly equivalent for long experimental channels that are predominantly laminar, 

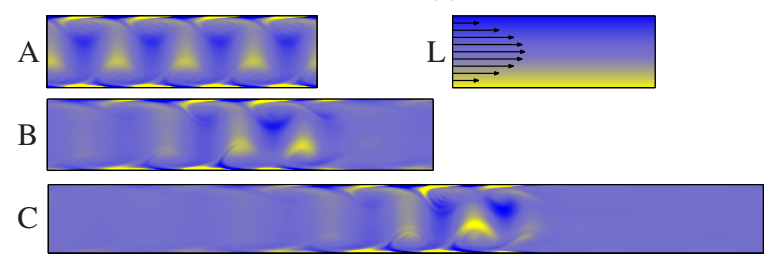

$\mathrm{D}$

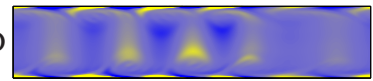

(a)

(b)

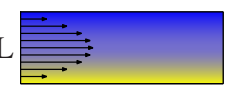

$\mathrm{E}$ (c)

(d)

FIGURE 1. (Color online) (a) Iso-vorticity contours of laminar flow (L), Tollmien Schlichting waves (A) and modulated Tollmien-Schlicting waves for different values of the parameters $k$ and $\operatorname{Re}(\mathrm{B}, \mathrm{C}$, D, E). Only perturbation on the laminar flow is represented except for L. Space-arclength parameter ( $d s \equiv \sqrt{d\|a\|^{2}+(d k)^{2}}$ ) diagram showing the evolution along (b) a MTSW branch taking $R_{5}$ UB TSWs to localisation at $R e=2800$, (c) an $R_{5}-R_{5}$ branch at $R e=2800$, and (d) a branch connecting $R_{4} \mathrm{UB}$ and $R_{5}$ LB TSWs at $R e=3000$. Coloring represents spanwise vorticity along a straight line at $y=-0.9$ and at time set by the crossing of the Poincaré section used in the solver.

but differ notably whenever the non-laminar (usually turbulent) fraction departs from close to zero. The massflow and the average pressure gradient over long distances remains constant in time, but the latter may fluctuate considerably over short distances as non-laminar fluid flows along. Numerically, only relatively short periodic domains can be simulated, so that a constant massflow must be enforced in order to properly capture the evolution of time-dependent solutions if these are to represent localised dynamics in very long, ideally infinite, domains (Mellibovsky \& Meseguer 2007). Merely travelling solutions have an exact representation in both constant pressure and constant-massflow PPF, while their stability and any time-dependent solution depends critically on the driving mechanism (Soibelman 1989; Barkley 1990; Soibelman \& Meiron 1991).

We define the Reynolds number for PPF as $R e=\frac{3 h U}{2 \nu}$, where $U$ is the mean streamwise velocity of the flow, which is held constant by adjusting $\Pi$ and corresponds to $2 / 3$ of the maximum velocity of the parabolic base flow. Taking $h$ and $3 U / 2$ as length and velocity scales, respectively, the basic laminar solution is a parabolic profile of purely streamwise velocity that only depends on the wall-normal coordinate $y$ (Figure 1aL): $\mathbf{u}_{0}=\left(1-y^{2}\right) \hat{\mathbf{x}}$.

We adopt the streamfunction formulation of Drissi et al. (1999), which naturally satisfies mass conservation and the no slip boundary condition at the walls, and at the same time preserves a constant massflow. The nondimensional streamfunction $\Psi(x, y ; t)$ is related to the nondimensional velocity field through $u=\partial_{y} \Psi$ and $v=-\partial_{x} \Psi$. The Navier-Stokes equations transform into

$$
\partial_{t} \nabla^{2} \Psi+\left(\partial_{y} \Psi\right) \partial_{x}\left(\nabla^{2} \Psi\right)-\left(\partial_{x} \Psi\right) \partial_{y}\left(\nabla^{2} \Psi\right)=\frac{1}{\operatorname{Re}} \nabla^{4} \Psi
$$

with streamwise periodic boundary conditions and $\partial_{y} \Psi(x, \pm 1 ; t)=\partial_{x} \Psi(x, \pm 1 ; t)=0$. The additional condition $\Psi(x, 1 ; t)-\Psi(x,-1 ; t)=4 / 3$ enforces the constant nondimensional mean axial velocity of $2 / 3$, while $\Psi(x,-1 ; t)=-2 / 3$ is chosen arbitrarily. The basic laminar flow reads $\Psi_{0}(y)=y\left(1-\frac{1}{3} y^{2}\right)$. In what follows, we consider the effect of finite perturbations $\widetilde{\Psi}(x, y ; t)$ to the basic solution:

$$
\Psi(x, y ; t)=\Psi_{0}(y)+\widetilde{\Psi}(x, y ; t)
$$

where $\widetilde{\Psi}$ is streamwise periodic and verifies homogeneous Neumann-Dirichlet boundary conditions at the walls: $\widetilde{\Psi}(x, \pm 1 ; t)=\partial_{x} \widetilde{\Psi}(x, \pm 1 ; t)=\partial_{y} \widetilde{\Psi}(x, \pm 1 ; t)=0$. Substituting (2.3) in (2.2) 
yields an equation equation for $\widetilde{\Psi}$ :

$$
\partial_{t} \nabla^{2} \widetilde{\Psi}=\mathbf{L}_{\operatorname{Re}}(\widetilde{\Psi})+\mathbf{N}(\widetilde{\Psi})
$$

with linear and nonlinear operators

$$
\left\{\begin{aligned}
\mathbf{L}_{\mathrm{Re}}(\widetilde{\Psi}) & =\frac{1}{\operatorname{Re}} \nabla^{4} \widetilde{\Psi}-\left(1-y^{2}\right) \partial_{x} \nabla^{2} \widetilde{\Psi}-2 \partial_{x} \widetilde{\Psi} \\
\mathbf{N}(\widetilde{\Psi}) & =\left(\partial_{x} \widetilde{\Psi}\right) \partial_{y} \nabla^{2} \widetilde{\Psi}-\left(\partial_{y} \widetilde{\Psi}\right) \partial_{x} \nabla^{2} \widetilde{\Psi}
\end{aligned}\right.
$$

\section{Numerical approach}

We use a Fourier-Legendre spectral expansion to approximate solutions of (2.4) in a domain $\Omega=\{(x, y) \in[0, \Lambda] \times[-1,1]\}:$

$$
\widetilde{\Psi}_{L M}(x, y, t)=\sum_{\ell=-L}^{L} \sum_{m=0}^{M} a_{\ell m}(t) \Psi_{\ell m}(x, y),
$$

where $\Psi_{\ell m}(x, y)=\mathrm{e}^{\mathrm{i} \ell k x} \Phi_{m}(y), \mathbf{a}=\left\{a_{\ell m}\right\}$ is the state vector with $a_{-\ell m}=a_{\ell m}^{*}$ ( $^{*}$ denotes complex conjugation), $k=2 \pi / \Lambda$ is the minimum streamwise wavenumber and $\Phi_{m}(y)=$ $\left(1-y^{2}\right)^{2} \mathrm{~L}_{m}(y)$, with $\mathrm{L}_{m}(y)$ the $m$-th Legendre polynomial. $\Phi_{m}( \pm 1)=\Phi_{m}^{\prime}( \pm 1)=0$ by construction, so that (3.1) identically satisfies homogeneous Dirichlet-Neumann boundary conditions at the walls.

Introducing the inner hermitian product of two arbitrary streamwise periodic fields $\zeta(x, y)$ and $\eta(x, y)$ in the domain $\Omega=[0, \Lambda] \times[-1,1]$ as

$$
(\zeta, \eta)_{\Omega}=\int_{0}^{\Lambda} \int_{-1}^{1} \zeta^{*} \eta \mathrm{d} y \mathrm{~d} x
$$

application of the spectral Galerkin scheme to (2.4) using (3.1) yields a system of ordinary differential equations for the expansion coefficients $a_{\ell m}(t)$ :

$$
\mathbb{A}_{p q}^{\ell m} \dot{a}_{\ell m}=\mathbb{B}_{p q}^{\ell m} a_{\ell m}+\mathbb{N}_{p q}(\mathbf{a})
$$

with

$$
\left\{\begin{aligned}
\mathbb{A}_{p q}^{\ell m} & =\left(\Psi_{p q}, \nabla^{2} \Psi_{\ell m}\right)_{\Omega}, \\
\mathbb{B}_{p q}^{\ell m} & =\left(\Psi_{p q}, \mathbf{L}_{\operatorname{Re}}\left(\Psi_{\ell m}\right)\right)_{\Omega}, \\
\mathbb{N}_{p q}(\mathbf{a}) & =\left(\Psi_{p q}, \mathbf{N}\left(\widetilde{\Psi}_{L M}\right)\right)_{\Omega} .
\end{aligned}\right.
$$

Summation over repeated indexes is assumed, and $(p, q) \in[0, L] \times[0, M]$. The nonlinear term $\mathbb{N}_{p q}(\mathbf{a})$ is evaluated pseudospectrally using Orszag's $3 / 2$ rule for dealiasing.

System (3.3) is advanced in time using a 4th order linearly-implicit backward differences scheme with 4th order extrapolation for the nonlinear term

$$
\begin{aligned}
(25 \mathbb{A}-12 \Delta t \mathbb{B}) \mathbf{a}^{(i+1)}= & \mathbb{A}\left(48 \mathbf{a}^{(i)}-36 \mathbf{a}^{(i-1)}+16 \mathbf{a}^{(i-2)}-3 \mathbf{a}^{(i-3)}\right)+ \\
& +\Delta t\left(48 \mathbb{N}^{(i)}-72 \mathbb{N}^{(i-1)}+48 \mathbb{N}^{(i-2)}-12 \mathbb{N}^{(i-3)}\right),
\end{aligned}
$$

where the superindex indicates time instant $\left(t_{i}=i \Delta t\right.$, with $\Delta t$ the time step). The time integration is started with an explicit 4th order Runge-Kutta method.

The timestep has been held constant at $\Delta t=10^{-2}$. A minimum of 50 Legendre polynomials and $7 \times n$ Fourier modes ( $n$ is the number of Tollmien-Schlichting waves that fit in the domain) in the wall-normal and streamwise spectral expansions, respectively, have been used throughout, but higher resolutions have been deployed for some extreme values of the parameters to ensure convergence. 
Travelling (or drifting) waves are characterised by a clear-cut streamwise wavelength $\lambda_{0}=$ $2 \pi / k_{0}$ ( $k_{0}$ is the fundamental wavenumber) and a travelling (phase) speed $c$. The state vector dependence on time simplifies to $a_{\ell m}(t)=a_{\ell m}^{\mathrm{TW}} \mathrm{e}^{-\mathrm{i} \ell k_{0} c t}$. Formal substitution in (3.3) leads to a nonlinear algebraic system of equations for the unknown speed $c$ and complex state vector $\mathbf{a}^{\mathrm{TW}}=\left\{a_{\ell m}^{\mathrm{TW}}\right\}$ :

$$
\left(\mathbb{B}_{p q}^{\ell m}+\mathrm{i} k_{0} \ell c \mathbb{A}_{p q}^{\ell m}\right) a_{\ell m}^{\mathrm{TW}}+\mathbb{N}_{p q}\left(\mathbf{a}^{\mathrm{TW}}\right)=0 .
$$

System (3.6) is completed with an extra condition on the phase of the solution to remove streamwise degeneracy and solved by means of a Newton-Krylov method (Kelley 1995) and solution branches are tracked in the $\left(\mathrm{Re}, k_{0}\right)$ parameter space using pseudoarclength continuation (Kuznetsov 2004).

When considered in a periodic domain of their wavelength, upper branch travelling waves typically undergo a series of superharmonic bifurcations into increasingly complex flows. When considered in longer domains, though, travelling wave trains may exhibit subharmonic instability.

Here we will be concerned with subharmonic instability of travelling waves, $i e$ instability to infinitesimal perturbations of wavelengths $\lambda=2 \pi / k$ (wavenumber $k$ ) longer than that of the wave. Perturbations of this type introduce a streamwise modulation to the wave train that may develop into localisation. In principle, $k$ can take any positive real value in the interval $[0,1]$. For $k / k_{0}=m / n \in \mathbb{Q}(n \in \mathbb{N}, m=1, \ldots, n)$, the instability of a travelling wave of wavenumber $k_{0}$ can be understood as the superharmonic instability of $n$ replicas of the travelling wave filling a periodic domain of length $\Lambda=n \lambda_{0}$ to periodic perturbations that fit $m$ times in the domain (Prat et al. 1998; Melnikov et al. 2014). Floquet theory analysis shows that modes with $m=j$ and $m=n-j$ are related by conjugation, so that only half the possible wavelengths need be explored. Furthermore, an analysis for a given $k / k_{0}$ can be performed on the minimal domain $n k_{0}$, such that $n / m$ expresses an irreducible fraction. Instability to irrational $k / k_{0}$ can only be interpreted in an infinitely long domain.

The representation of $a_{\ell^{\prime} m}^{\mathrm{TW}}$ in the $n$-fold replicated domain $\Omega_{n}=[-1,1] \times\left[0, n \lambda_{0}\right], n \in \mathbb{N}$ is

$$
a_{\ell m}(t)=a_{\ell m}^{\mathrm{TW}_{n}} \mathrm{e}^{-\mathrm{i} \ell k_{n} c t}
$$

where $k_{n}=k_{0} / n$ and $a_{\ell m}^{\mathrm{TW}}=a_{\ell^{\prime} m}^{\mathrm{TW}}$ for $\ell=n \ell^{\prime}$ and 0 otherwise. Linear stability is then analysed by considering infinitesimal disturbances to the travelling wave:

$$
a_{\ell m}(t)=\left(a_{\ell m}^{\mathrm{Tw}_{n}}+\varepsilon_{\ell m} \mathrm{e}^{\sigma t}\right) \mathrm{e}^{-\mathrm{i} \ell k_{n} c t} .
$$

Substituting (3.8) in (3.3) and neglecting terms beyond linear results in the generalised eigenproblem

$$
\left(\sigma-\mathrm{i} k_{n} \ell c\right) \mathbb{A}_{p q}^{\ell m} \varepsilon_{\ell m}=\mathbb{B}_{p q}^{\ell m} \varepsilon_{\ell m}+D_{\mathbf{a}} \mathbb{N}_{p q}^{\ell m}\left(\mathbf{a}^{\mathrm{TW}_{n}}\right) \varepsilon_{\ell m}
$$

which can be solved for eigenvalues $\sigma_{i}$ and eigenvectors $\epsilon_{i}=\left\{\varepsilon_{\ell m_{i}}\right\} . D_{\mathbf{a}} \mathbb{N}_{p q}^{\ell m}\left(\mathbf{a}^{\mathrm{TW}_{n}}\right)$ is the linearisation of $\mathbb{N}$ about $\mathbf{a}^{\mathrm{TW}_{n}}$ and $(p, q)=[0, n L] \times[0, M]$. For small $n$ (3.9) can be solved using direct eigenvalue methods such as QZ. For large resolutions, Arnoldi iteration is required in combination with adequate Cayley transformations (Trefethen \& Bau 1997).

A comprehensive analysis would incur unaffordable computational time-scales, so that the focus has been mainly set on the subset of $k$ 's that can be analysed in domains of up to $6 \lambda_{0}$, which are sufficiently long to initiate a streamwise modulation that effectively localises when followed to longer domains. As a matter of fact, previous subharmonic stability analyses of travelling waves in PPF (Drissi et al. 1999) reveal oscillatory Hopf bifurcations from which emanate branches of streamwise modulated wave trains (Jimenez 1990) that are time-periodic when observed from a reference frame moving at the average streamwise speed of the waves.

To compute and track modulated waves beyond their region of stability, we have applied Newton-Krylov and pseudoarclength continuation to the nonlinear system of equations resulting 

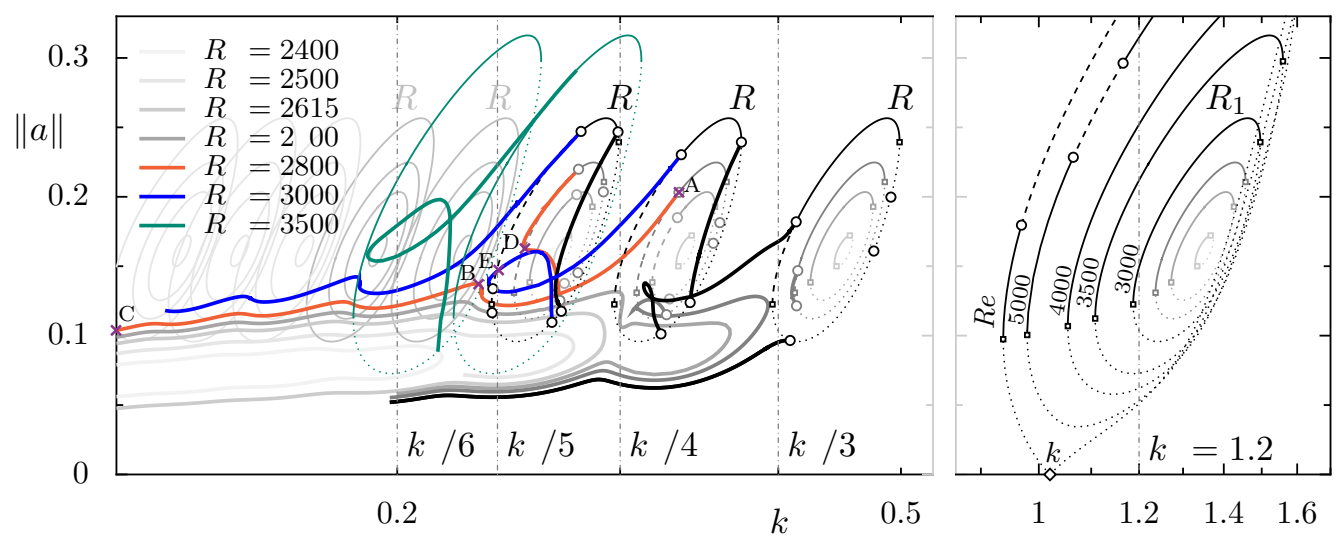

FIgURE 2. (Color online) Amplitude of TSW and MTSW as a function of $k$. The number of Fourier modes was adapted during $k$-continuation to ensure sufficient streamwise resolution. Re number is color-coded. Thick lines represent MTSW. Continuous, dashed and dotted thin lines labelled $R_{n}$ denote subharmonically stable UB, unstable UB and LB trains of $n$ TSW solutions. Open squares are saddle-node and open circles Hopf bifurcation points. The diamond indicates the subcritical bifurcation of TSWs from the base flow.

from root finding for the map defined by consecutive crossings of a Poincaré section $\mathbb{P}$ :

$$
\mathbf{a} \mapsto \tilde{\mathbf{a}}=\mathbb{P}(\mathbf{a})=\mathbb{T}(\Delta z) \Phi^{T}(\mathbf{a}),
$$

where $\Phi^{t}$ is the flow generated by (3.3) and $[\mathbb{T}(\Delta z) \mathbf{a}]_{\ell m}=\exp (-\mathrm{i} \ell k \Delta z) a_{\ell m}$ is the shift operator. $T$ (the modulation period) and $\Delta z$ (the streamwise drift) satisfy

$$
\begin{aligned}
(\tilde{\mathbf{a}}-\mathbf{a}) \cdot \hat{\mathbb{T}}(\mathbf{a}) & =0 \\
(\tilde{\mathbf{a}}-\mathbf{a}) \cdot \dot{\mathbf{a}} & =0
\end{aligned}
$$

where $[\hat{\mathbb{T}}(\mathbf{a})]_{\ell m}=-\mathrm{i} \ell k a_{\ell m}$ is the group orbit tangent and $\dot{\mathbf{a}}=\mathbb{A}^{-1}[\mathbb{B} \mathbf{a}+\mathbb{N}(\mathbf{a})]$ the rate of change of the state vector. The orthogonality conditions (3.11) simultaneously define the Poincaré section and remove the drift degeneracy along the group orbit.

\section{Results and discussion}

Although linearly stable for Reynolds numbers up to $R e_{c}=5772.22$ (Orszag 1971), the basic flow is subcritically unstable to well known streamwise periodic drifting waves that go by the name of Tollmien-Schlichting waves (TSW, figure 1aA and online movie). The critical wavenumber is $k_{c}=1.02056$.

When considered at constant $R e<R e_{c}$ (Figure 2, $R_{1}$ ), TSWs appear as disconnected closed loops with a limited range of existence $k_{0} \in\left[k_{0_{\min }}(R e), k_{0_{\max }}(R e)\right]$, such that within these limits two solutions exist for any given $k$ that may be classified as either upper (UB, continuous and dashed lines) or lower (LB, dotted) branch. We have followed the steps of (Jimenez 1990; Pugh \& Saffman 1988) in computing the superharmonic stability of TSWs to validate our numerics. Lower branch TSWs are mere saddles, while upper-branch TSWs, which are initially stable, undergo a series of transitions into increasingly complex flows that results in chaotic dynamics well before $R e_{c}$ is reached (Jimenez 1990) (see the unstable regions -dashed lines- of $R_{1}$ in figure 2). When considered in longer domains, though, TSW trains exhibit subharmonic instability.

For the subharmonic stability we have systematised the analysis of Drissi et al. (1999) to explore up to $R e=3000$ in domains of length $\Lambda=\left(2 \pi / k_{0}\right) n$ with $n \leqslant 6 \in \mathbb{N}$. Figure 2 
represents a normalised measure of the amplitude of the solution $\left(\|\mathbf{a}\|=\left[\sum_{\ell m}\left|a_{\ell m}\right|^{2}\right]^{1 / 2}\right)$ against the extent of the periodic domain as measured by the smallest wavenumber $(k=2 \pi / \Lambda)$ it can accommodate. Thus, the TSW family $R_{1}$ appears replicated in figure 2 at $k=k_{0} / n$ for $n \in \mathbb{N}$, each replica representing a train of $n$ TSWs that fits exactly once in the domain under consideration ( $R_{n}$ TSW solution, standing for $n$ replicas).

At large enough $R e$ and low enough $k$, TSW iso-Re curves start intersecting (see fading $R_{n}$ families in figure 2), indicating that the same periodic domain can take various TSW trains including different number of replicas of the appropriate wavelength. The subharmonic bifurcation analysis results in a set of curves in $R e-k$ parameter space that reduce to bifurcation points when considered at constant $R e$ (open circles). These points, together with the saddle-node points, divide each iso- $R e$ loop of TSWs into segments with a different count of unstable eigenmodes. A distinction has been made between the unstable LB (dotted), the stable part of the UB (solid) and, if present, the subharmonically unstable portions of the UB (dashed).

All subharmonic instabilities happen to be of the Hopf type, such that emanating solutions incorporate a modulational frequency in addition to the degenerate frequency associated to the streamwise solid body motion of the TSW. We have continued the modulated TollmienSchlichting waves (MTSW) to analyse the nonlinear evolution of the streamwise modulation resulting from the subharmonic instability. The family of MTSWs has a convoluted dependence on the parameters that hinders a simple interpretation, which is resolved by analysing one parameter at a time.

Some of the constant-Re branches take from a train of a certain number $n$ of TSWs to another train with the same number of TSWs with an interim of a mild temporal modulation that introduces a group velocity on top of the phase velocity of the TSW. Figure 1c recreates one such continuation, for a $R e=2800$ branch of MTSWs connecting with $R_{5}$ at both ends (short orange line in figure 2), by picturing the evolution of vorticity on a streamwise section at $y=-0.9$ as progress is made along the continuation curve. The maximum departure from steadiness is labelled D and vorticity contours are shown in figure 1aD.

Other branches evolve into localisation. Figure $1 \mathrm{~b}$ shows the branch issued, also at $R e=2800$, from the instability of a slightly longer TSW (figure 1aA) to perturbations of a shorter wavelength that modulates trains of 4 replicas $\left(R_{4}\right)$. The localised solution of figure 1ac (online movie) shares key features with puffs and spots in other shear flows: while the leading edge modifies the laminar flow in the midplane, the trailing edge has a tendency to attach to the walls. The process that leads to the localised solution is nevertheless non-monotonic, as weak TSWs try to grow within the quasi-laminar space left inbetween separating replicas. Traces of these weak TSWs appear as fading colored bands Figure $1 \mathrm{~b}$ and manifest themselves in figure 2 (long orange line) as failed attempts of the branch to loop back to reconnect with a longer train of TSWs. The first attempt of reconnection is labelled B and shown in figure 1aB (online movie). The MTSWs labelled B and D in figure 1 correspond to turns of the continuation branches facing each other in figure 2 (orange lines). The snapshots, shown in figure 1a, indeed bear some resemblance to one another, which indicates proximity. As a matter of fact, the two branches approach at these points as $R e$ is increased, collide, and separate into two new branches at $R e=3000$, one that localises from $R_{5}$ and the other that connects $R_{4}$ to $R_{5}$ (blue lines). This latter branch shows a tendency to localise, as evidenced in figure 1d by the widening gray region halfway of the continuation, but rectifies in a loop to end up incorporating a new replica of TSWs. The snapshot labelled $\mathrm{E}$ and depicted in figure $1 \mathrm{aE}$ is in fact related with $\mathrm{B}$ and $\mathrm{D}$ through a codimension-two bifurcation point somewhere around $(k, R e) \sim(0.3,2900)$. This trend is corroborated at $R e=3500$ (green lines), where the branch localising from $R_{5}$ at $R e=3000$ has already evolved into a loop connecting $R_{5}$ to $R_{6}$.

It might be argued that, unlike the localised MTSWs studied here, streamwise localised solutions in other shear flows are not patterned (Mellibovsky et al. 2009; Duguet et al. 2009), 


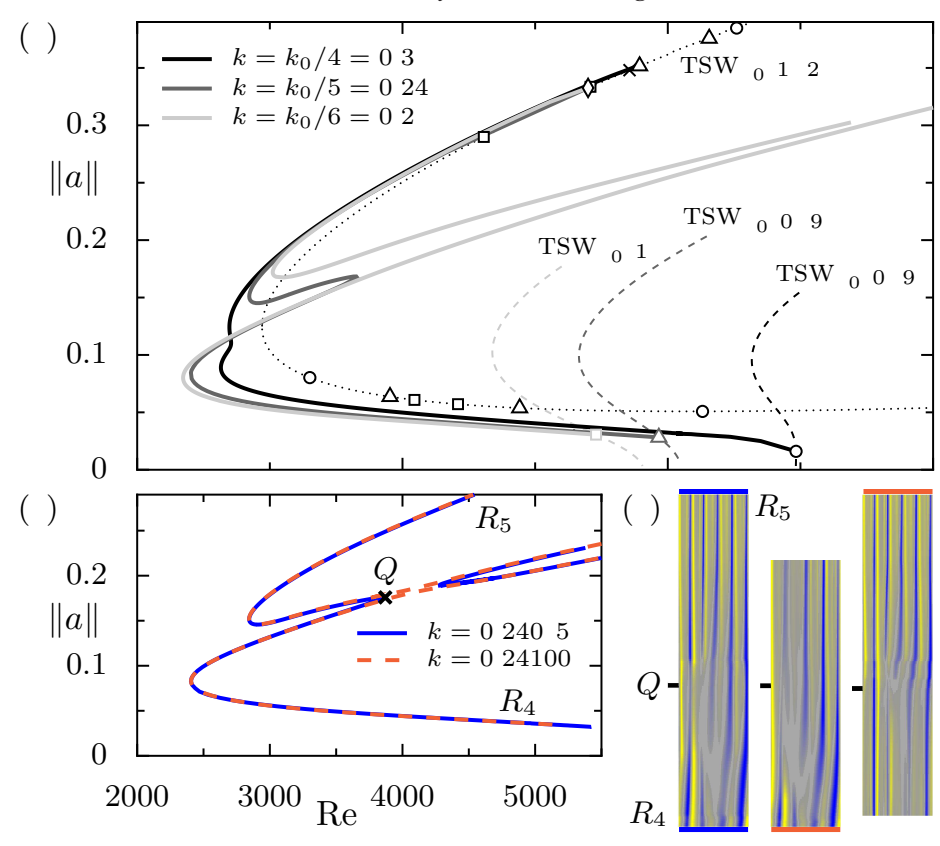

FIgURE 3. (Color online) (a) Re-continuation of MTSWs (solid lines; at $k$ values as indicated in figure 2) compatible with subharmonic modulation of the $k=1.2 \mathrm{TSW}$ (dotted) and their connections with LB TSWs. Circles, triangles, squares and diamonds represent $n=3,4,5,6$ subharmonic Hopf bifurcations, respectively. Irrelevant MTSW have not been plotted for clarity. The cross marks the first superharmonic Hopf bifurcations. The scale and labels for the x-axis are shared with those of panel b. (b) MTSWs at neighboring values of $k$ and (c) corresponding space-arclength diagrams. $R_{4}$ and $R_{5}$ denote connections to TSWs, while $Q$ indicates solutions closely related by a codimension-2 point.

so that the localisation mechanism might be fundamentally different from the one we describe. However, there is evidence that pipe Poiseuille localised solutions (Avila et al. 2013; Chantry et al. 2014) are indeed patterned, except that the wavy structure is in fact masked by the rather short modulational envelope. Chaotic sets developed from extended versions of these solutions clearly exhibit wave trains with a varying count of wave periods embedded (Ritter et al., to be published). This will most probably be the case for localised plane Couette and plane Poiseuille solutions too (Brand \& Gibson 2014; Zammert \& Eckhardt 2014). The non-snaking localisation mechanism (Burke \& Knobloch 2006) found in double-diffusive convection (Beaume et al. $2013 b$ ) cannot apply to plane Couette, plane Poiseuille or pipe flows, as the required condition that a nontrivial streamwise-independent state exists is not fulfilled by any of these flows.

It is clear from $k$-continuation that MTSWs connect both with upper and lower branch TSWs, and how this comes about when smoothly varying $R e$ at constant $k$ is vital to putting the snaking hypothesis to test. The simplest approach to the issue is to select a TSW with fixed $k_{0}$ as a reference and restrict the $R e$-continuation of MTSW branches to domains with $k=k_{0} / n, n \in$ $3,4,5,6 \ldots$. We have chosen $k_{0}=1.2$ to have some of the continuation branches cut right through the loops (see figure 2). Figure 3a shows $R e$-continuation branches for $k=k_{0} / 4, k_{0} / 5$ and $k_{0} / 6$. The $k=k_{0} / 4$ branch (continuous black line) connects seemingly uneventfully a LB $R_{3}$ with an UB $R_{4}$. The $k_{0} / 5$ branch (dark gray), which cuts right through a $k$-continuation loop, seems to hint at a snaking-like process by incorporating a back and forth detour in the otherwise uneventful connection of a LB $R_{4}$ and an UB $R_{5}$. The $k_{0} / 6$ branch (light gray), however, while still connecting with a LB $R_{5}$, challenges the pattern by neither connecting with an UB, nor even hinting an approach to connect at higher $R e$. 
( )

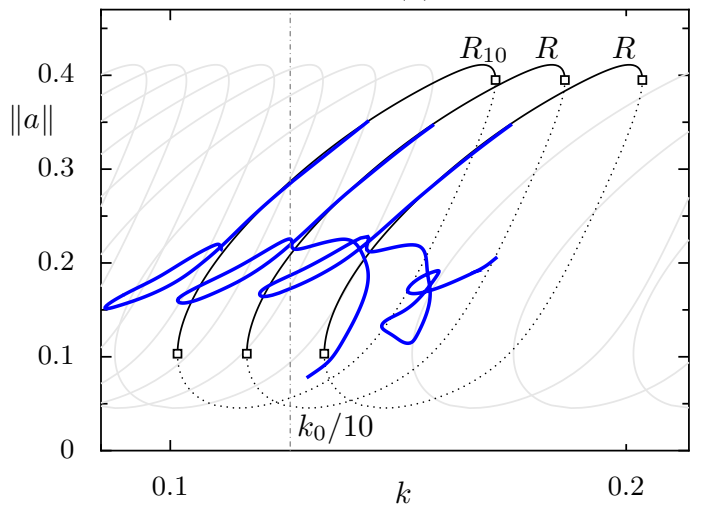

$($ )

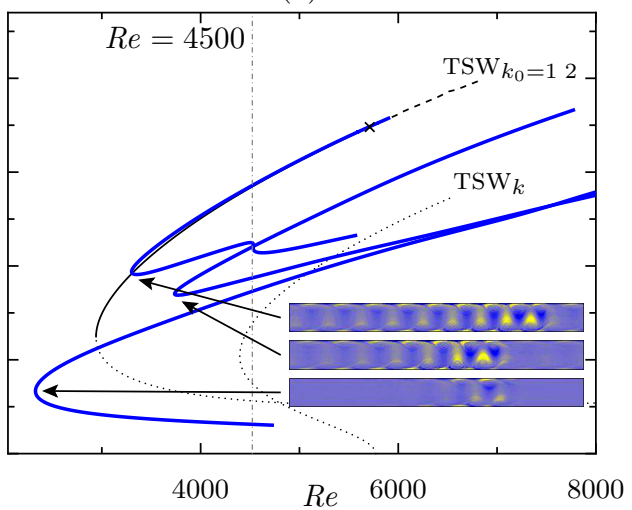

FIGURE 4. (Color online) (a) $k$-continuation of MTSWs at $R e=4500$ issued from subharmonic instability of $R_{8}, R_{9}$ and $R_{10}$ TSWs. (b) Re-continuation of MTSWs at $k=k_{0} / 10=0.12$. The cross marks the first superharmonic Hopf bifurcation. The snapshots correspond to the saddle-nodes of the three disconnected branches of MTSWs. The dash-dotted lines in (a) and (b) indicate the cross-section depicted in (b) and (a), respectively.

Although for some particular moderate values of $k, R e$-continued branches of MTSWs bend back and forth in a snaking-like fashion connecting different trains of TSWs that fit in the same domain, the accumulation process that is typical of spanwise localisation is not robust. Figure $3 \mathrm{~b}$,c shows how small variations in $k$ disrupt the continuous single branch (continuous blue line), which breaks in two separate branches (dashed orange) that reach high $R e$ values, possibly infinity. While both continuations evolve parallely away from LB $R_{4}$ TSWs, one manages to incorporate a new replica $(k=0.24075)$ and connect to $R_{5}$, while the other keeps the modulation and a certain localisation for increasing $\operatorname{Re}(k=0.24100)$. For large enough domains, it appears utterly improbable that a localised MTSW will robustly accumulate the number of bends that are required before connection to a full train of UB TSWs is effective. Instead, most, if not all, of the bends will have broken up producing disconnected families of localised MTSWs of different lengths. The $k=0.2$ upper lobe in Fig. 3a (light gray) is a clear example of how disconnected families of solutions with a different modulated wave number count can arise in the same domain. In longer domains this will result in disconnected localised solutions of different lengths. Even when one of these bends exists, there also exists a disconnected branch of MTSWs that appears in a saddle-node at a higher $R e$ and faces the saddlenode of the bend. It is precisely the collision of these two saddle-nodes upon smoothly varying $k$ that disrupts the snaking mechanism. This is reminiscent of the blending of separate regions of regular and semi-infinite snaking into a unique region of semi-infinite snaking observed for some parameter values of the Swift-Hohenberg equation (Burke \& Knobloch 2006). Furthermore, the existence of a conserved quantity, which in our case is mass-flux, accounts for the slanted arrangement of the leftmost saddle-nodes (Dawes 2008; Beaume et al. 2013a), thus allowing for localised solutions to exist at lower and higher values of the parameter than is possible in regular snaking. Physically, this means that while the multiplicity of spanwise localised solutions of different lengths is restricted to a finite $R e$-range (where the bends exist, within the broadended Maxwell point), a surging number of streamwise localised solutions of varying length extending to high $R e$ appears as longer domains are considered.

Figure 4a illustrates the accumulation process of loops at $R e=4500$ and $k$ around $1.2\left(R_{10}\right)$. The leftmost loop in figure 2 (green line) at $R e=3500$ has stretched and, as Tsws get cluttered in longer domains at higher $R e$, continuation loops of MTSWs start overlapping in $k$. Each loop implies a disconnection of MTSW branches when continued in $R e$, such that three lobes are 
expected for $k=k_{0} / 10=0.12$ in figure $4 \mathrm{~b}$. As a matter of fact, the domain size chosen cuts right through the rightmost end of the loop, which indicates that the upper lobe is just forming. For $k$ slightly lower, the three lobes are perfectly established. This completes the picture of the intricate accumulation process. As the domain size is increased, an upper branch forms from a longer train of shorter TSWs that combines with the uppermost existing lobe as the rightmost part of the iso-Re loop is encountered. Meanwhile, the pre-existing lobes get compressed and the lowest two connect and merge as the leftmost side of the loop is crossed (as shown in figure $3 b$ ). All in all, as loops accumulate, the creation of new lobes at the top is faster than the merging of lobes at the bottom and an increasing number of disconnected branches coexist as longer domains are considered. The snapshots in figure 4 correspond to the saddle-nodes of the various lobes. It is clear that longer localised MTSWs, embedding a higher count of TSW replicas, are encountered as higher lobes are considered. All these solutions coexist beyond large enough $R e$ in sufficiently long domains.

Symmetries and the nature of the existing nontrivial extended solutions play a key role in homoclinic snaking. Regular snaking results from stationary front pinning to the wave train underlying the localised solution (Burke \& Knobloch 2006). Homogeneous extended solutions give rise to non-snaking behaviour as the fronts can pin to the underlying solution regardless of its spatial extent but only so for values of the governing parameter corresponding to Maxwell points (Burke \& Knobloch 2006; Beaume et al. 2013b). No such behaviour can be expected in the streamwise direction of any of the usual shear flows, as all secondary solutions are patterned. Regarding symmetries, the abscense of space reversibility due to the fore-aft broken symmetry of Poiseuille flow, introduces significant modifications to the snaking scenario as demonstrated for simple models such as the Swift-Hohenberg equation (Burke et al. 2009). Among the predicted changes, we have observed the obvious drift of the localised solutions, but no such thing as the disconnection of snakes and ladders into isolas has been detected. Although snaking has originally been discovered and studied in variational systems, it is a well known fact that the problem needs not be variational in time to produce this type of behaviour, as demonstrated for dissipative systems such as plane Couette flow (Schneider et al. 2010). Finally, the introduction of a large scale neutral mode (Dawes 2008), associated to the existence of a conservation law, results in a substantial enlargement of the region of existence of localised states and the snaking branches becomes slanted. Two-dimensional PPF conserves massflux (or, alternatively, mean pressure gradient), which justifies the slanted arrangement of the leftmost saddle-nodes even if snaking has been disrupted and isolated branches of localised solutions have emerged.

\section{Concluding remarks}

We have adopted a streamfunction formulation for two-dimensional flows and introduced a set of purposely developed numerical methods to solve and track in parameter space both travelling and modulated travelling wave solutions, as well as to compute their linear stability.

We have used these methods to dissect the mechanism for shear flow streamwise localisation through its analysis in the paradigmatic case of two-dimensional PPF in order to unveil its resemblance yet at the same time essential differences with spanwise localisation. While its emergence follows subharmonic instability of a periodic wave, the snaking mechanism whereby a long spatial modulation develops into a localising envelope that confines a shrinking wave packet via the successive loss of wave replicas, decisively breaks down. As a result, while multiplicity of spanwise localised solutions of different lengths is restricted to a limited $R e$ range, in very long domains branches of arbitrarily long streamwise localised solutions -their length necessarily quantised- extend to high $R e$, thus potentially contributing to the formation and growth of a chaotic saddle that may sustain turbulent transients. We have found evidence, in a much longer domain with $k=0.08$, of phenomena such as quasiperiodicity and chaos, 
a precursor to turbulence, as well as splitting of localised spots. A thorough analysis of the mechanisms behind these observations is underway but beyond the scope of the present study.

In simple models featuring snaking, localised solutions can be understood as homoclinic orbits of the spatial ordinary differential equation that results from dropping the time derivative (Kirchgässner 1982; Budd \& Kuske 2005). These homoclinic orbits take from the base flow (a spatial fixed point) back to the base flow, with a natural number of intervening quasi-orbits around the nontrivial patterned flow (a spatial periodic orbit), hence the qualifier homoclinic with which the word snaking is commonly prefixed. In the limit, a front can be interpreted as an heteroclinic orbit joining the base state and the patterned state. This simple interpretation does not apply to localised solutions in shear flows such as plane Poiseuille or pipe Poiseuille (at least not straightforwardly), as the modulational nature of solutions precludes stationary front pinning, such that time-dependence cannot be eliminated from the partial differential equation. Recent studies in our same setup of two-dimensional PPF suggest that at high Reynolds numbers where turbulence expands, the analysis of laminar-turbulent interfaces may require a specific treatment (Teramura \& Toh 2015).

We acknowledge financial support from grants FIS2013-40674-P and 2014SGR1515 from the Spanish and Catalan governments, respectively.

\section{REFERENCES}

Avila, K., Moxey, D., De Lozar, A., Avila, M. \& Hof, B. 2011 The onset of turbulence in pipe flow. Science 333 (6039), 192-196.

Avila, M., Mellibovsky, F., Roland, N. \& Hof, B. 2013 Streamwise-localized solutions at the onset of turbulence in pipe flow. Phys. Rev. Lett. 110 (22), 224502.

BARKLEY, D. 1990 Theory and predictions for finite-amplitude waves in 2-dimensional plane Poiseuille flow. Phys. Fluids A 2 (6), 955-970.

Beaume, C., Bergeon, A., Kao, H.S \& Knobloch, E. 2013a Convectons in a rotating fluid layer. J. Fluid Mech. 717, 417-448.

Beaume, C., Knobloch, E. \& Bergeon, A. 2013b Nonsnaking doubly diffusive convectons and the twist instability. Phys. Fluids 25 (11), 114102.

Bergeon, A., Burke, J., Knobloch, E. \& Mercader, I. 2008 Eckhaus instability and homoclinic snaking. Phys. Rev. E 78 (4), 046201.

BRAND, E. \& GiBSON, JF. 2014 A doubly localized equilibrium solution of plane Couette flow. J. Fluid Mech. 750 (R3).

BUDD, C.J. \& KUSKE, R. 2005 Localized periodic patterns for the generalized Swift-Hohenberg equation. Phys. D 208, 73-95.

Burke, J., Houghton, S.M. \& KNOBloch, E. 2009 Swift-Hohenberg equation with broken reflection symmetry. Phys. Rev. E 80 (3), 036202.

Burke, J. \& KnObloch, E. 2006 Localized states in the generalized Swift-Hohenberg equation. Phys. Rev. $E 73(5), 056211$.

Burke, J. \& KNOBlOCH, E. 2007 a Homoclinic snaking: Structure and stability. Chaos 17 (3), 037102.

BuRKE, J. \& KNOBLOCH, E. 2007b Snakes and ladders: Localized states in the Swift-Hohenberg equation. Phys. Lett. A 360 (6), 681-688.

Chantry, M., \& Kerswell, R. R. 2015 Localization in a spanwise-extended model of plane Couette flow. Phys. Rev. E 91 (4).

Chantry, M., Willis, A. P. \& Kerswell, R. R. 2014 Genesis of streamwise-localized solutions from globally periodic traveling waves in pipe flow. Phys. Rev. Lett. 112 (16).

Chen, T.S. \& Joseph, D.D. 1973 Subcritical bifurcation of plane Poiseuille flow. J. Fluid Mech. 58, 337-351.

Cross, Mark C \& Hohenberg, Pierre C 1993 Pattern formation outside of equilibrium. Rev. Modern Phys. 65 (3), 851.

DAWES, J.H.P. 2008 Localized pattern formation with large-scale mode: slanted snaking. SIAM J. Appl. Dyn. Sys. 7 (1), 186-206. 
Drissi, A., NET, M. \& MERCADER, I. 1999 Subharmonic instabilities of Tollmien-Schlichting waves in two-dimensional Poiseuille flow. Phys. Rev. E 60 (2), 1781-1791.

Duguet, Y., Schlatter, P. \& Henningson, D.S. 2009 Localized edge states in plane Couette flow. Phys. Fluids 21 (11), 111701.

Duguet, Y., Willis, A.P. \& Kerswell, R.R. 2008 Transition in pipe flow: the saddle structure on the boundary of turbulence. J. Fluid Mech. 613, 255-274.

Eckhardt, B., Schneider, T.M., Hof, B. \& Westerweel, J. 2007 Turbulence transition in pipe flow. Ann. Rev. Fluid Mech. 39, 447-468.

Faisst, H. \& ECKhardT, B. 2003 Travelling waves in pipe flow. Phys. Rev. Lett. 91 (22), 224502.

GIBSON, JF. \& BRAND, E. 2014 Spanwise-localized solutions of planar shear flows. J. Fluid Mech. 745, 25-61.

Hamilton, J.M., Kim, J. \& WalefFe, F. 1995 Regeneration mechanisms of near-wall turbulence structures. J. Fluid Mech. 287, 317-348.

Hof, B., van Doorne, C.W.H., Westerweel, J., Nieuwstadt, F.T.M., Faisst, H., Eckhardt, B., Wedin, H., Kerswell, R.R. \& WalefFe, F. 2004 Experimental observation of nonlinear travelling waves in turbulent pipe flow. Science 305 (5690), 1594-1598.

ITANO, T. \& TOH, S. 2000 The dynamics of bursting process in wall turbulence. J. Phys Soc Jpn 70 (3), 703-716.

JimenEZ, J. 1990 Transition to turbulence in 2-dimensional Poiseuille flow. J. Fluid Mech. 218, 265-297.

KELlEY, C. T. 1995 Iterative Methods for Linear and Nonlinear Equations. SIAM.

KERSWELL, R. R. \& TUTTY, O. R. 2007 Recurrence of travelling waves in transitional pipe flow. J. Fluid Mech. 584, 69-102.

KIRCHGäSSNER, K. 1982 Wave solutions of reversible problems and solutions. J. Differ. Equations 45 (1), $113-127$.

Kreilos, T., Veble, G., Schneider, T.M. \& Eckhardt, B. 2013 Edge states for the turbulence transition in the asymptotic suction boundary layer. J. Fluid Mech. 726, 100-122.

KuZnetsov, Y. A. 2004 Elements of Applied Bifurcation Theory, 3rd edn. Springer.

MellibovsKy, F. \& ECKHARDT, B. 2012 From travelling waves to mild chaos: a supercritical bifurcation cascade in pipe flow. J. Fluid Mech. 709, 149-190.

Mellibovsky, F. \& Meseguer, A. 2007 Pipe flow transition threshold following localized impulsive perturbations. Phys. Fluids 19 (4), 044102.

Mellibovsky, F., Meseguer, A., Schneider, T.S. \& Eckhardt, B. 2009 Transition in localized pipe flow turbulence. Phys. Rev. Lett. 103 (5), 054502.

Melnikov, K., Kreilos, T. \& ECKhardT, B. 2014 Long-wavelength instability of coherent structures in plane Couette flow. Phys. Rev. E 89 (4), 043008.

Mercader, I., Batiste, O., Alonso, A. \& Knobloch, E. 2009 Localized pinning states in closed containers: Homoclinic snaking without bistability. Phys. Rev. E 80 (2), 025201.

NAGATA, M. 1990 3-dimensional finite-amplitude solutions in plane Couette-flow - bifurcation from infinity. J. Fluid Mech. 217, 519-527.

ORSZAG, S.A. 1971 Numerical simulation of incompressible flows with simple boundaries - accuracy. J. Fluid Mech. 49, 75-.

Prat, J., Mercader, I. \& Knobloch, E. 1998 Resonant mode interactions in Rayleigh-Bénard convection. Phys. Rev. E 58 (3), 3145-3156.

Pugh, J.D. \& SAFFMAN, P.G. 1988 Two-dimensional superharmonic stability of finite-amplitude waves in plane Poiseuille flow. J. Fluid Mech. 194, 295-307.

REYNOLDS, O. 1883 An experimental investigation of the circumstances which determine whether the motion of water shall be direct or sinuous and of the law of resistance in parallel channels. Phil. Trans. Roy. Soc. Lond. 174, 935-982.

Ritter, P., F., Mellibovsky. \& Avila, M. , to be published Origin of spatiotemporal fluctuations in pipe flow .

Schneider, T.M., Gibson, J.F. \& Burke, J. 2010 Snakes and ladders: localized solutions of plane Couette flow. Phys. Rev. Lett. 104 (10), 104501.

Schneider, T.M., Gibson, J.F., Lagha, M., De Lillo, F. \& ECKhardt, B. 2008 Laminar-turbulent boundary in plane Couette flow. Phys. Rev. E 78 (3), 037301.

Solbelman, I. 1989 A study of finite amplitude bifurcations in plane Poiseuille flow. PhD thesis, California Institute of Technology, Pasadena, CA, USA. 
Soibelman, I. \& Meiron, D.I. 1991 Finite-amplitude bifurcations in plane Poiseuille flow - 2dimensional Hopf-bifurcation. J. Fluid Mech. 229, 389-416.

Teramura, T. \& ToH, S. 2015 Chaotic self-sustaining structure embeded in turbulent-lamina interface. arXiv:1503.04551.

Trefethen, Lloyd N. \& BAU, DAVID 1997 Numerical Linear Algebra. SIAM.

WEDIN, H. \& KERSWELL, R.R. 2004 Exact coherent structures in pipe flow: travelling wave solutions. J. Fluid Mech. 508, 333-371.

Wygnanski, I.J. \& Champagne, F.H. 1973 On transition in a pipe. Part 1. The origin of puffs and slugs and the flow in a turbulent slug. J. Fluid Mech. 59, 281-335.

Zahn, J.P., Toomre, J., Spiegel, E.A. \& Gough, D.O. 1974 Nonlinear cellular motions in Poiseuille channel flow. J. Fluid Mech. 64, 319-345.

ZAMMERT, S. \& ECKHARDT, B. 2014 Streamwise and doubly-localised periodic orbits in plane poiseuille flow. J. Fluid Mech. 761, 348-359. 